\title{
Accuracy of MSCT coronary angiography with 64-slice technology: first experience
}

\author{
Sebastian Leschka ${ }^{1}$, Hatem Alkadhi ${ }^{1}$, André Plass ${ }^{2}$, Lotus Desbiolles ${ }^{1}$, Jürg Grünenfelder ${ }^{2}$, \\ Borut Marincek ${ }^{1}$, and Simon Wildermuth ${ }^{1 *}$ \\ ${ }^{1}$ Department of Medical Radiology, Institute of Diagnostic Radiology, University Hospital Zurich, Rämistrasse 100, 8091 \\ Zurich, Switzerland; and ${ }^{2}$ Clinic for Cardiovascular Surgery, University Hospital Zurich, Switzerland
}

Received 17 December 2004; revised 7 March 2005; accepted 15 March 2005; online publish-ahead-of-print 19 April 2005

See page 1451 for the editorial comment on this article (doi:10.1093/eurheartj/ehi322)

\section{KEYWORDS}

Computed tomography;

Conventional coronary

angiography;

Coronary artery disease

\section{Introduction}

Invasive coronary angiography (ICA) is the accepted reference standard for the assessment of coronary artery stenoses because of its unprecedented temporal and spatial resolution and the ability to perform therapeutic interventions in the same session. Although the associated risk for serious complications is small, ${ }^{1}$ the inconvenience for the patient and economic deliberations have strengthened the search for a non-invasive alternative. Current multi-slice computed tomography (MSCT) scanners provide promising results in the assessment of coronary artery disease (CAD), but some segments are not evaluative because of motion artifacts or severe vessel wall calcification. ${ }^{2-4}$ The recently developed 64-slice CT scanner generation provides $0.4 \mathrm{~mm}$ nearly isotropic voxels in a rotation time of $0.37 \mathrm{~s}$, thus increasing temporal and spatial resolution when compared with previous CT scanner types. This is accomplished by using periodic motion of the focal spot in the longitudinal direction resulting in two simultaneously acquired overlapping projections of 32 detectors (z-Sharp Technology)..$^{5}$

The aim of our study was to investigate the accuracy of 64-slice CT for assessing haemodynamically significant stenoses of the coronary arteries in comparison with ICA.

\footnotetext{
*Corresponding author. Tel: +411255 3662; fax: +4112554443.

E-mail address: simon.wildermuth@usz.ch
}

\section{Methods}

\section{Study population}

Seventy-three consecutive patients ( 54 male, 19 female; mean age $62.0 \pm 10.8$ years; range $34-82$ years) were referred to our centre between October 2004 and January 2005 for suspected CAD or prior to coronary artery bypass surgery. Exclusion criteria for CT were as follows: previous allergic reactions to iodine contrast media, severe renal insufficiency (creatinine level, $>120 \mu \mathrm{mol} / \mathrm{L}$ ), haemodynamic instability, atrial fibrillation, inability to follow breath-hold commands, previous percutaneous transluminal coronary stent placement, and previous bypass surgery. Seven patients were excluded because of elevated creatinine levels $(n=4)$, previous stent placement $(n=2)$, or disclaimed to take part in the study $(n=1)$. The remaining 67 patients (50 male, 17 female; mean age $60.1 \pm 10.5$ years; range $34-82$ years) underwent both ICA and CT coronary angiography and were included in this study. The mean interval time between ICA and CT was $14.1 \pm 14.5$ days (median 13; range 1-55 days). Twenty-four (36\%) investigations were performed in patients presenting with signs of unstable angina pectoris, $43(64 \%)$ patients were examined before undergoing coronary artery bypass surgery. From the 67 patients, 40 $(60 \%)$ had taken $\beta$-receptor-blocking as part of their baseline medication at the time of the CT examination. The study was approved by our institutional ethics committee and all participating patients gave written informed consent.

\section{Invasive coronary angiography}

ICA was performed according to standard techniques and multiple views were stored on a CD-ROM. The angiograms were evaluated by an experienced observer who was blinded to the results from 
CT coronary angiography. The coronary arteries were segmented according to the guidelines of the American Heart Association ${ }^{6}$ and to a previous publication ${ }^{4}$ as follows: the right coronary artery (RCA) was subdivided in a proximal, middle, and distal parts; the posterior descending artery was considered as independent because of the variability of its origin; the left circumflex artery (LCX) was divided into proximal and distal parts; the left anterior descending artery (LAD) was divided into proximal, middle, and distal parts; the diagonal (DIA) branches, obtuse marginal (OM) branches, and posterolateral (PLA) branches were considered as being independent segments. Each vessel segment was scored as being significantly stenosed, defined as a diameter reduction of $>50 \%$ or not. Coronary artery analysis was performed in all vessels with a diameter down to $1.5 \mathrm{~mm}$, including those vessels distal to complete occlusions.

\section{CT coronary angiography}

All CT scans were performed on a 64-slice scanner with a $0.37 \mathrm{~s}$ rotation time (Somatom Sensation 64, Siemens, Forchheim, Germany). A bolus of $80 \mathrm{~mL}$ iodixanol (Visipaque $320 \mathrm{mg} / \mathrm{mL}$, Amersham Health, Buckinghamshire, UK) was injected into an antecubital vein at a flow rate of $5 \mathrm{~mL} / \mathrm{s}$, followed by a $50 \mathrm{~mL}$ saline chasing bolus. Start delay was defined by bolus tracking in the ascending aorta and scan start was automatically initiated $5 \mathrm{~s}$ after reaching the threshold [140 HU (HU, Hounsfield Units)]. After this, scanning was performed from the tracheal bifurcation to the diaphragm using the following parameters: X-ray tube potential $120 \mathrm{kV}$, effective tube current $680 \mathrm{~mA}$, slice collimation $64 \times 0.6 \mathrm{~mm}^{2}$, table feed $9.2 \mathrm{~mm} /$ rotation, and pitch 0.24 . All CT scans were performed using an implemented fully automated realtime anatomy based dose regulation (CARE Dose 4D). The overall scan time was shorter than $12 \mathrm{~s}$ (median $11.2 \mathrm{~s}$, range 10.7-11.9 s) and the mean total time for the examination was $<15 \mathrm{~min}$ (median $12 \mathrm{~min}$, range 8-14 $\mathrm{min}$ ). We used retrospective electrocardiographic (ECG) gating for optimal heart phase selection. ${ }^{7}$

The implemented adaptive cardio volume approach was used for data reconstruction. Depending on the heart rate throughout the examination, axial slices were reconstructed synchronized to the ECG by a single sector ( $\leq 65$ b.p.m) or two sector algorithm (>65 b.p.m) using data from one or two consecutive heartbeats. ${ }^{5}$ When necessary, R-wave indicators were manually repositioned to improve the quality of synchronization. Images were reconstructed at $10 \%$ intervals of the cardiac cycle to allow assessment of coronary arteries at that cardiac phase with minimal vessel motion. Slices with a thickness of $0.75 \mathrm{~mm}$ (increment $0.5 \mathrm{~mm}$ ) and a medium soft-tissue reconstruction kernel (B30f) were used for evaluating coronary arteries.

\section{CT data analysis}

Coronary artery analysis was performed using the same guidelines ${ }^{6}$ as for ICA. Two independent and blinded observers without knowledge of the patients clinical history, the indication for the patients referral, and the results from ICA classified each vessel segment interactively on a post-processing workstation (Second Wizard, Siemens, Forchheim, Germany) for the presence of haemodynamically significant stenoses. Similar to ICA, significant stenosis was defined as narrowing of the coronary lumen exceeding $50 \%$ and all vessels with a diameter down to $1.5 \mathrm{~mm}$ including those vessels distal to complete occlusions were included in the analysis. The vessel diameters were measured on reconstructions perpendicularly oriented to the vessel course. Depending on the individual coronary anatomy and image quality, different visualization techniques such as multi-planar reformations (MPR), maximum intensity projections (MIP), and volume rendering (VR) were used. Each vessel was analysed on at least two planes, one parallel, and one perpendicular to the course of the vessel. In case of disagreement between the two observers, a final decision was obtained by consensus.
Image quality was classified for each segment as being excellent (no artifacts, unrestricted evaluation), good (minor artifacts, good diagnostic quality), adequate (moderate artifacts, acceptable for routine clinical diagnosis), and poor/not evaluative (severe artifacts impairing accurate evaluation). When image quality rating was not excellent, the reasons for impaired visualization were selected from the following list: low vessel opacification, calcium deposits or opacified adjacent structures (such as contrastenhanced ventricle or cardiac vein), and motion artifacts. Motion artifacts were defined as any impairment of image quality caused by vessel movement resulting in blurred or doubled vessel contours. Arterial wall calcification was rated as either moderate (small isolated wall calcifications) or massive (extensive wall calcification with beam hardening artifacts impairing arterial lumen visualization).

\section{Statistical analysis}

Statistical analysis was performed using a commercially available statistical software (SPSS 11.5 for Windows, SPPS Incorporation, Chicago, IL, USA). Quantitative variables are expressed as mean \pm standard deviation. Inter-observer agreements were expressed as Cohen's kappa statistics ${ }^{8}$ and interpreted by the guidelines of Landis and Koch. ${ }^{9}$ Sensitivity, specificity, positive predictive value, and negative predictive value for 64-slice CT coronary angiography for detecting significant stenoses in all coronary arteries were calculated from $\chi^{2}$ tests of contingency, and 95\% confidence intervals $(\mathrm{Cl})$ were calculated from binomial expression. Because of the possible interdependencies between different vessel segments, the statistics were calculated on a vessel-based analysis. ICA was regarded as the standard of reference.

\section{Results}

Using ICA, a total of 176 haemodynamically significant stenoses, $17 \%$ of them $(30 / 176)$ being complete vessel occlusions, were identified in $70 \%(47 / 67)$ of the patients. Onevessel disease was present in 6\% (4/67), two-vessel disease in $15 \%(10 / 67)$, and three-vessel disease in $49 \%(33 / 67)$. Significant coronary artery stenosis was absent in $30 \%$ (20/67).

No additional $\beta$-blockers were administered prior to the CT examination resulting in a mean heart rate of $66.3 \pm 14.7$ beats per minute (range 48-90). Regarding CT coronary angiography, kappa values for image quality, calcifications, and stenosis detection were $0.88,0.87$, and 0.95 , respectively, indicating a good to excellent inter-observer agreement. Results for coronary artery stenosis detection, image quality, calcifications, reasons for impaired image quality, and diagnostic accuracy in each segment and the results regarding proximal, middle, and distal vessel segments (including side branches) are summarized in Table 1.

Image quality was rated as being excellent in $61 \%$ $(612 / 1005)$, good in $31 \%(310 / 1005)$, and adequate in $8 \%$ $(83 / 1005)$ of all segments with the best results in the proximal coronary arteries. No vessel segment had to be excluded from analysis. Calcifications were present in $49 \%$ $(494 / 1005)$ of all coronary branches, $36 \%$ of them $(180 /$ 494) being referred to as massive calcifications causing beam hardening artifacts decreasing lumen visualization. Consequently, the most common cause for impaired image quality resulted from severely calcified vessel walls (56\%; 229/393). Motion artifacts were recognized in $5 \%$ $(51 / 1005)$ of the coronary vessels in $24 \%$ of the patients $(16 / 67)$. Of these patients $(13 / 16), 81 \%$ had heart rates $\geqslant 75$ beats per minute (median 78 per minute, range 


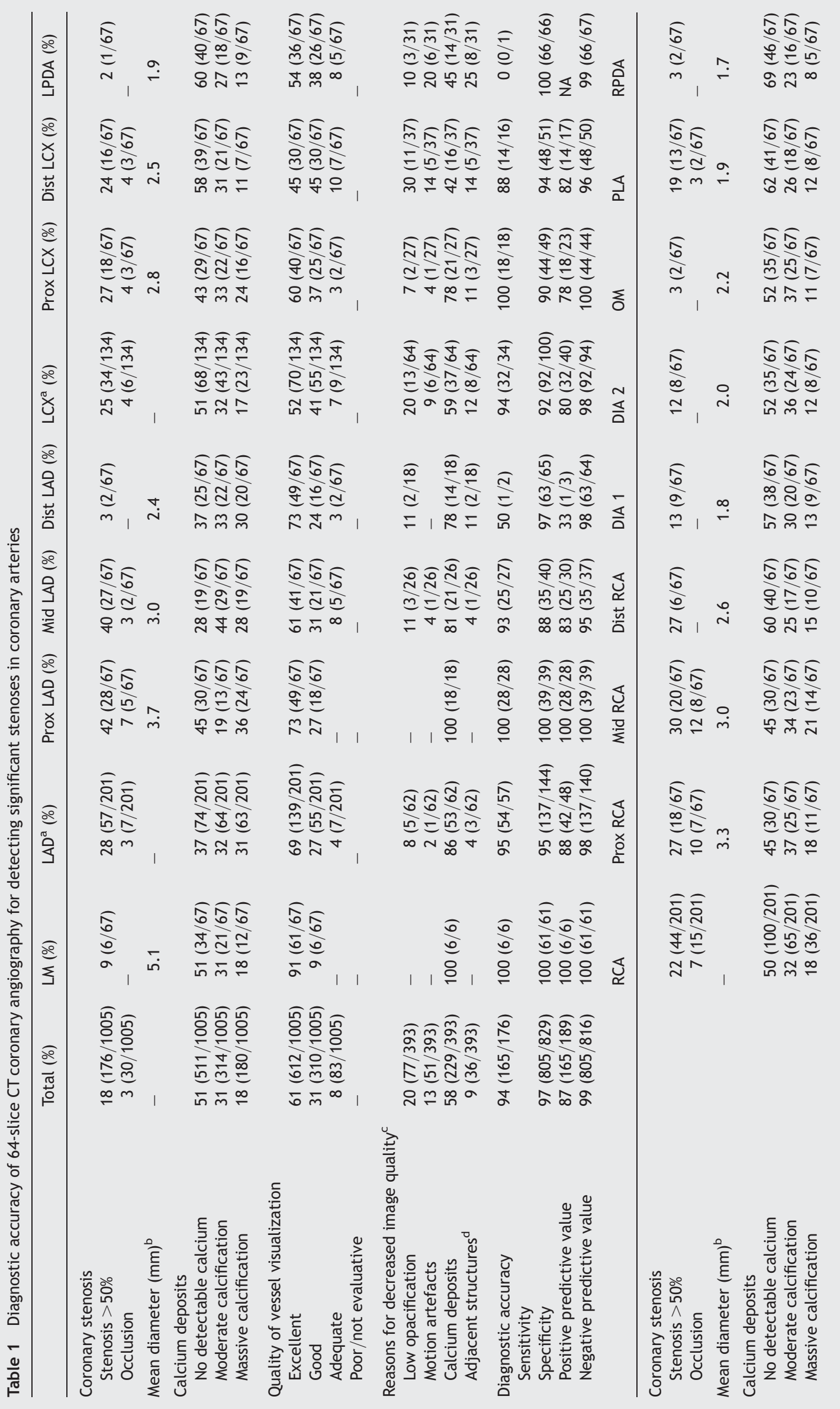




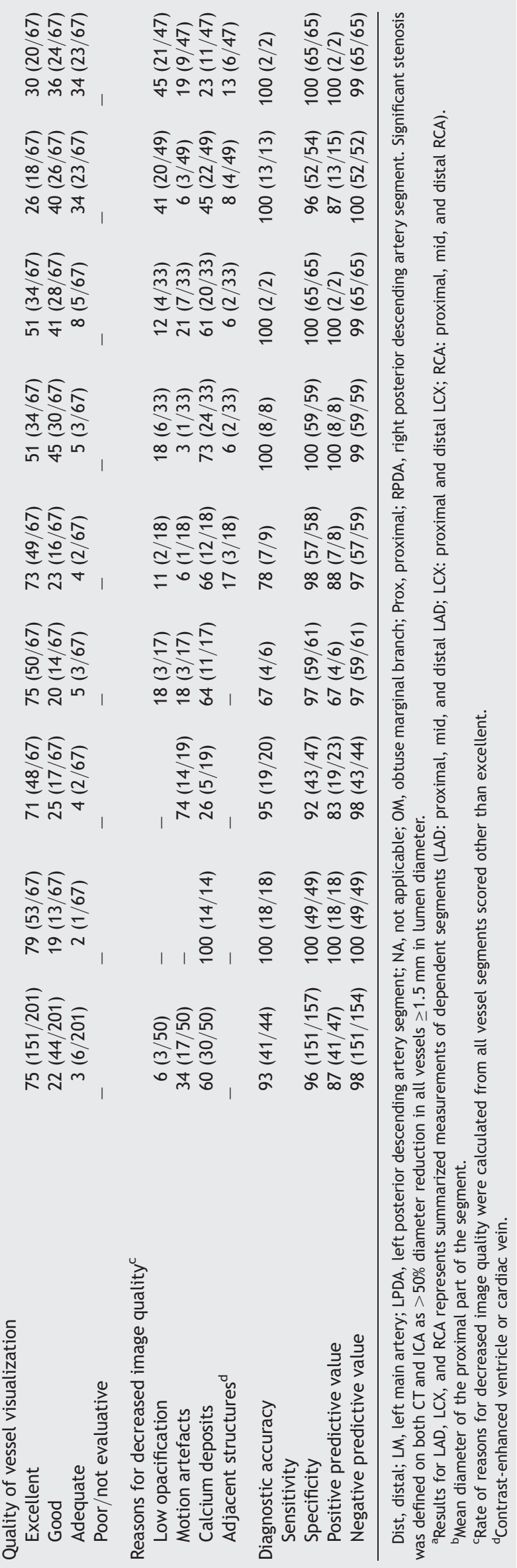

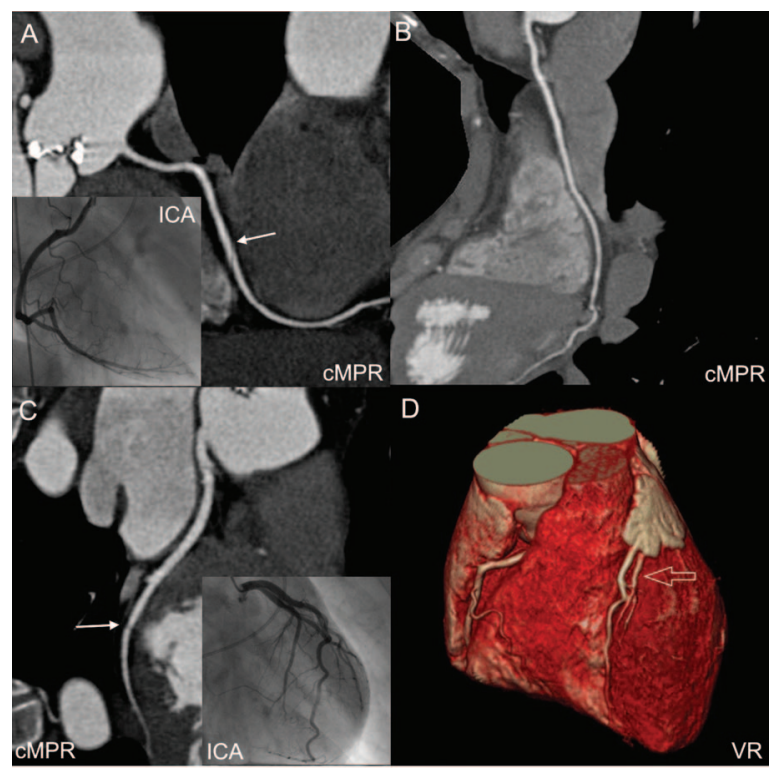

Figure 1. Normal coronary arteries with 64-slice CT coronary angiography and ICA in a 74-year-old woman admitted for suspected CAD. Curved multiplanar reformations (CMPR) of the RCA $(A)$, LAD $(B)$, and LCX (C) demonstrate no stenosis. Some slight motion artifacts are present in mid-RCA and distal LCX (arrows), but image quality was rated as good in those segments. VR image $(D)$ delineates the three-dimensional course of the coronary arteries and also presenting venous opacification by not disturbing artery visualization (open arrow). Corresponding ICA demonstrates normal right (Inlay in $A$ ) and left coronary arteries (Inlay in $C$ ).

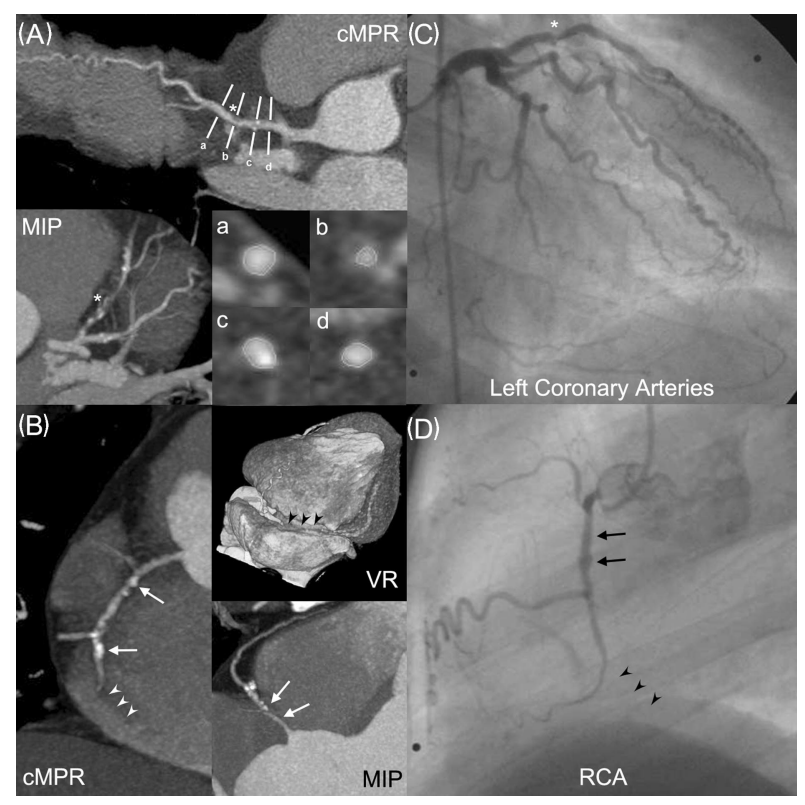

Figure 2. Sixty-four-slice CT coronary angiography and ICA in a 44-year-old man with atypical chest pain. CMPR $(A)$, MIP, and perpendicular cut-planes (Inlays a-d) delineate multiple non-calcified plaques in mid-LAD, one leading to a haemodynamically significant stenosis (asterisk, cut-plane b). In the RCA, CMPR $(B)$, VR image, and MIP demonstrate multiple non-significant wall irregularities in the mid-segment (arrows) and occlusion of the distal segment (arrowheads). As shown in the ICA ( $C$ and $D)$, all lesions were correctly identified.

67-90). Most motion artifacts affected the mid-RCA (28\%; $14 / 51), 71 \%$ of these patients $(10 / 14)$ had elevated heart rates $\geqslant 75$ beats per minute (median 80 per minute, range 70-90). However, of the 51 observed motion artifacts, 47 
led to an only slight impairment of image quality with the vessel being still evaluative. In the four segments with motion artifacts degrading the image quality to adequate, two were responsible for false-negative ratings. A case with typical motion artifacts is demonstrated in Figure 1. Low vessel opacification was found in $\sim 8 \%(77 / 1005)$ of the coronary arteries, most of them in the PLA $(30 \%$; $20 / 67)$, and the RPDA (31\%; 21/67). Overlaying adjacent structures were recognized less frequently as cause of decreased image quality $(4 \%$; $36 / 1005)$. In all cases, both reasons lead to an only slight degradation of image quality from excellent to good.

From a total of 176 haemodynamically significant stenoses detected with ICA, 165 were correctly recognized with CT coronary angiography. The overall sensitivity was $94 \%$ $(165 / 176 ; 95 \% \mathrm{Cl}: 90.2-96.2)$, the specificity was $97 \%$ (805/829; 95\% Cl: 96.4-97.6), the positive predictive value was $87 \%(165 / 189 ; 95 \% \mathrm{Cl}: 84.0-89.6)$, and the negative predictive value was $99 \%(805 / 816 ; 95 \% \mathrm{Cl}$ : 97.9-99.2). Three lesions were missed because of motion artifacts: one in the mid-RCA, one in the distal LCX, and one in the posterior descending artery. Depending on the grade of diameter reduction, severely calcified lesions were judged as being either stenotic or non-stenotic. Eight lesions were missed because of severe wall calcification: two in the distal RCA, two in the mid-LAD, one in the distal LAD, two in the first diagonal branch, and one in the distal LCX. Twenty-four lesions observed with CT coronary angiography were falsepositive and all were located in severely calcified vessel segments: four in mid-RCA, two in the distal RCA, five in mid$\angle A D$, two in distal $L A D$, one in the first diagonal branch, five in the proximal LCX, three in distal LCX, and two in the PLA branch. Figure 2 demonstrates an example for coronary artery stenosis detection by MSCT and ICA.

Regarding a patient-based analysis, all patients without CAD could be correctly identified with CT. In all patients with CAD at least one significant lesion was correctly classified with $\mathrm{CT}$.

\section{Discussion}

To become a clinically accepted tool for the examination of patients with suspected $C A D$, the main requisite for $C T$ coronary angiography includes the complete visualization of all therapeutic relevant coronary arteries without excluding segments. ${ }^{3}$ With four-slice CT, a sensitivity of $58-86 \%$ for the detection of coronary stenosis has been reported, ${ }^{10-14}$ but up to $32 \%$ of the vessels had to be excluded from analysis because of a decreased image quality. ${ }^{10}$ Using 16-slice CT, overall sensitivity including all segments was reported to be between 73 and $95 \%$, depending on the diameter of the segment, the modality of analysis, and the patient selection criteria. $^{2-4,15}$ However, in some studies evaluation was limited to branches having a diameter $>2 \mathrm{~mm} .^{3,15} \mathrm{In}$ this study, we evaluated all arteries being $>1.5 \mathrm{~mm}$ in diameter, and no segment had to be excluded from analysis, thereby finding a sensitivity of $94 \%$ and a specificity of $97 \%$ for the detection of significant coronary stenoses. Because of different patient populations and imaging protocols compared with the earlier mentioned studies, a direct comparison of study results is not permitted. Nevertheless, the data reported herein using a 64-slice CT scanner suggest a certain improvement regarding diagnostic accuracy. The high negative predictive value of $99 \%$ in this study furthermore suggests an important future role of CT coronary angiography for reliably excluding CAD in patients with an equivocal clinical presentation, who may currently undergo a cost-extensive ICA. ${ }^{16}$

Recent studies have reported two major limitations for reliable assessment of all coronary segments: motion artifacts and severe coronary calcifications. ${ }^{2-4}$ Regarding motion artifacts, no additional $\beta$-blockers prior to the CT examination were administered in this study resulting in a heart rate between 48 and 89 beats per minute. By selecting several reconstruction intervals relative to the cardiac cycle, it was possible to compensate for motion artifacts in the majority of affected vessels by individually choosing the phase with minimal vessel motion for each segment. In general, image quality was decreased with higher heart rates, but only two significant lesions were missed because of severe heart motion. Regarding coronary calcifications, half of the coronary segments in this study contained wall calcifications, $18 \%$ being severe calcifications causing beam hardening artifacts with a decrease in lumen visualization. Calcium deposits were found to be responsible for eight false-negative and all 24 false-positive judgments with CT. In addition, all false-positive lesions also appeared in ICA as wall irregularities.

Reflecting our experience with cardiac 16-slice CT, improved spatial and temporal resolution of the new scanner generation increases image quality and facilitates the assessment of CAD. Moreover, the scanning time is shortened to $<12 \mathrm{~s}$, allowing a decreased breath-hold time, a better exploitation of contrast-media with less enhancement of adjacent structures, and a lower dose of applied contrast media.

We acknowledge the following limitations of this study. First, some specific segments (e.g. PLA branches and posterior descending artery) harboured only a low number of significant stenoses. Secondly, nearly two-thirds of the study patients were referred prior to coronary artery bypass surgery presenting with a high rate of severe wall calcifications and three-vessel disease. Therefore, patient selection bias may possibly limit the transfer of these results to clinical practice. Especially, the assumption that MSCT angiography can reliably rule out CAD cannot be demonstrated on the basis of the results with this high CAD prevalence cohort. Furthermore, this setting with patients having a high pre-test probability of the disease may have resulted in an overestimation of the ability of MSCT to detect and to rule out stenoses. Thirdly, no quantitative coronary angiography was performed and stenoses were semi-quantitatively assessed with both ICA and MSCT. This method of stenosis evaluation may have been influenced by a subjectivity bias. However, a kappa value of 0.95 corresponds to an excellent inter-observer agreement and may argue against such a subjectivity bias. On the other hand, such a high inter-observer agreement may be difficult to be obtained in a cohort of patients with a lower prevalence of CAD. Next, the radiation exposure inherent with $\mathrm{CT}$ may be a limitation of the technique. The most effective way to reduce radiation exposure is prospective ECG tube current modulation (ECG pulsing). ${ }^{17}$ We did not apply this technique because of a possible impairment of image quality during early-diastole, a phase which is often crucial when evaluating the RCA. To reduce 
radiation exposure, instead of quantitative calcium scoring on additional non-contrast scans, we descriptively assessed arterial wall calcifications on CT coronary angiography. In addition, the implemented anatomy based dose regulation software significantly reduces effective radiation doses. ${ }^{18}$ Finally, MSCT and ICA were not performed simultaneously, but the median delay between the two test was small with regard to the natural progression of CAD. ${ }^{19}$

In conclusion, our initial results suggest that 64-slice CT allows a non-invasive assessment of haemodynamically significant CAD with a high diagnostic accuracy. Extensive arterial wall calcifications still impair vessel assessment, but no segment had to be excluded from analysis. Further prospective studies have to analyse the threshold of vessel size and heart rate sensible for reliable assessment of coronary artery stenosis, and should aim in examining patient populations with a low pre-test probability of CAD in whom catheterization could be avoided.

\section{Acknowledgement}

This research has been supported by the National Center of Competence and Research, Computer Aided and Image Guided Medical Interventions (NCCR CO-ME) of the Swiss National Science Foundation.

\section{References}

1. Smith SCJ, Dove JT, Jacobs AK, Kennedy JW, Kereiakes D, Kern MJ, Kuntz RE, Popma JJ, Schaff HV, Williams DO, Gibbons RJ, Alpert JP, Eagle KA, Faxon DP, Fuster V, Gardner TJ, Gregoratos G, Russell RO, Smith SCJ. ACC/AHA guidelines for percutaneous coronary intervention (revision of the 1993 PTCA guidelines)-executive summary: a report of the American College of Cardiology/American Heart Association task force on practice guidelines (Committee to revise the 1993 guidelines for percutaneous transluminal coronary angioplasty) endorsed by the Society for Cardiac Angiography and Interventions. Circulation 2001;103:3019-3041.

2. Ropers D, Baum U, Pohle K, Anders K, Ulzheimer S, Ohnesorge B, Schlundt C, Bautz W, Daniel WG, Achenbach S. Detection of coronary artery stenoses with thin-slice multi-detector row spiral computed tomography and multiplanar reconstruction. Circulation 2003;107:664-666.

3. Mollet NR, Cademartiri F, Nieman K, Saia F, Lemos PA, McFadden EP, Pattynama PM, Serruys PW, Krestin GP, de Feyter PJ. Multislice spiral computed tomography coronary angiography in patients with stable angina pectoris. J Am Coll Cardiol 2004;43:2265-2270.

4. Martuscelli E, Romagnoli A, D’Eliseo A, Razzini C, Tomassini M, Sperandio M, Simonetti G, Romeo F. Accuracy of thin-slice computed tomography in the detection of coronary stenoses. Eur Heart J 2004;25:1043-1048.
5. Flohr T, Stierstorfer K, Raupach R, Ulzheimer $\mathrm{S}$, Bruder $\mathrm{H}$. Performance evaluation of a 64-slice CT system with z-flying focal spot. Rofo 2004;176:1803-1810.

6. Austen WG, Edwards JE, Frye RL, Gensini GG, Gott VL, Griffith LS, MCGoon DC, Murphy ML, Roe BB. A reporting system on patients evaluated for coronary artery disease. Report of the ad hoc committee for grading of coronary artery disease, council on cardiovascular surgery, American Heart Association. Circulation 1975;51:5-40.

7. Ohnesorge B, Flohr T, Becker C, Knez A, Kopp AF, Fukuda K, Reiser MF. Cardiac imaging with rapid, retrospective ECG synchronized multilevel spiral CT. Radiologe 2000;40:111-117.

8. Cohen J. A coefficient of agreement for nominal scales. Educ Psychol Meas 1960;20:37-46.

9. Landis JR, Koch GG. The measurement of observer agreement for categorical data. Biometrics 1977;33:159-174.

10. Achenbach S, Giesler T, Ropers D, Ulzheimer S, Derlien H, Schulte C, Wenkel E, Moshage W, Bautz W, Daniel WG, Kalender WA, Baum U. Detection of coronary artery stenoses by contrast-enhanced, retrospectively electrocardiographically-gated, multislice spiral computed tomography. Circulation 2001;103:2535-2538.

11. Kopp AF, Schroeder S, Kuettner A, Baumbach A, Georg C, Kuzo R, Heuschmid M, Ohnesorge B, Karsch KR, Claussen CD. Non-invasive coronary angiography with high resolution multidetector-row computed tomography. Results in 102 patients. Eur Heart J 2002;23:1714-1725.

12. Nieman K, Oudkerk M, Rensing BJ, van Ooijen P, Munne A, van Geuns RJ, de Feyter PJ. Coronary angiography with multi-slice computed tomography. Lancet 2001;357:599-603.

13. Achenbach S, Ulzheimer S, Baum U, Kachelriess M, Ropers D, Giesler T, Bautz W, Daniel WG, Kalender WA, Moshage W. Noninvasive coronary angiography by retrospectively ECG-gated multislice spiral CT. Circulation 2000;102:2823-2828.

14. Becker CR, Knez A, Leber A, Treede H, Ohnesorge B, Schoepf UJ, Reiser MF. Detection of coronary artery stenoses with multislice helical CT angiography. J Comput Assist Tomogr 2002;26:750-755.

15. Nieman K, Cademartiri F, Lemos PA, Raaijmakers R, Pattynama PM, de Feyter PJ. Reliable noninvasive coronary angiography with fast submillimeter multislice spiral computed tomography. Circulation 2002;106:2051-2054.

16. Schoepf UJ, Becker CR, Ohnesorge BM, Yucel EK. CT of coronary artery disease. Radiology 2004;232:18-37.

17. Trabold T, Buchgeister M, Kuttner A, Heuschmid M, Kopp AF, Schroder S, Claussen $C D$. Estimation of radiation exposure in 16-detector row computed tomography of the heart with retrospective ECG-gating. Rofo Fortschr Geb Rontgenstr Neuen Bildgeb Verfahr 2003;175:1051-1055.

18. Greess $H$, Nomayr A, Wolf $H$, Baum $U$, Lell M, Bowing B, Kalender W, Bautz WA. Dose reduction in CT examination of children by an attenuation-based on-line modulation of tube current (CARE Dose). Eur Radiol 2002;12:1571-1576.

19. Kondos GT, Hoff JA, Sevrukov A, Daviglus ML, Garside DB, Devries SS, Chomka EV, Liu K. Electron-beam tomography coronary artery calcium and cardiac events: a 37-month follow-up of 5635 initially asymptomatic low- to intermediate-risk adults. Circulation 2003;107:2571-2576. 